\title{
ELABORAÇÃO E AVALIAÇÃO DE UM QUESTIONÁRIO PARA DIAGNÓSTICO DO GERENCIAMENTO DE RESÍDUOS DE SERVIÇOS DE SAÚDE
}

\author{
Tatiane Bonametti Veiga ${ }^{1}$
}

Silvano da Silva Coutinho ${ }^{2}$

Angela Maria Magosso Takayanagui ${ }^{3}$

\begin{abstract}
RESUMO
O presente trabalho teve como objetivo descrever o processo de construção de um questionário para avaliação da geração e gerenciamento de resíduos de serviços de saúde em Instituição de Ensino Superior. A elaboração desse questionário foi fundamentada nas diretrizes apresentadas pelo Conselho Nacional do Meio Ambiente e pela Agência Nacional de Vigilância Sanitária, sendo observadas as orientações da Associação Brasileira de Normas Técnicas e os princípios presentes na Política Nacional de Resíduos Sólidos. O questionário contempla a geração e as diferentes fases do manejo interno e externo de resíduos, sendo construído junto a especialistas e avaliado por juízes da área. É importante salientar que esse questionário pode ser utilizado para realizar um diagnóstico inicial em Instituições de Ensino a fim de verificar sua situação perante aos resíduos gerados em suas delimitações. Esse diagnóstico é fundamental para auxiliar na elaboração e acompanhamento do Plano de Gerenciamento de Resíduos de Saúde tanto em Instituições de Ensino como outros órgãos responsáveis pela geração de resíduos de serviços de saúde.
\end{abstract}

PALAVRAS-CHAVE: Instituições de Ensino Superior; Questionários; Resíduos de Serviços de Saúde

\section{DEVELOPMENT AND EVALUATION OF A QUESTIONNAIRE FOR DIAGNOSIS OF MEDICAL WASTE MANAGEMENT}

\begin{abstract}
This study aimed to describe the process of developing a questionnaire to evaluate the generation and management of medical waste in Higher Education Institution. The development of this questionnaire was based on the guidelines presented by the National Environmental Council and the National Health Surveillance Agency, and also the guidelines of the Brazilian Association of Technical Standards and of the National Policy on Solid Waste. The questionnaire includes the generation and different stages of the internal and external waste management, being built with experts and evaluated by professional judges. The questionnaire built from this study can be used to make an initial diagnosis in education

\footnotetext{
${ }^{1}$ Doutora em Ciências, Programa de Pós-graduação em Enfermagem em Saúde Pública, Universidade de São Paulo - USP. Engenheira Civil. tati.veiga@yahoo.com.br.

${ }^{2}$ Doutor em Ciências, Programa de Pós-graduação em Enfermagem em Saúde Pública, Universidade de São Paulo - USP. Professor Adjunto da Universidade do Centro Oeste do Paraná.

silvano.unicentro@gmail.com.br.

${ }^{3}$ Doutora em Enfermagem. Universidade de São Paulo - USP. Professora Associada da Escola de Enfermagem de Ribeirão Preto (EERP/USP). amtakay@eerp.usp.br.
} 
institutions in order to verify their status related to the waste generated in their boundaries. This diagnosis is crucial to assist in the development and monitoring of the Medical Waste Management Plan in both education institutions and other departments responsible for generation of medical waste. KEY-WORDS: Higher Education Institutions; Questionnaires; Medical Waste.

\section{ELABORACIÓN Y EVALUACIÓN DE UN CUESTIONARIO PARA DIAGNÓSTICO DE GESTIÓN DE RESIDUOS SANITARIOS}

\section{RESUMEN}

Este estudio objetivó describir el proceso de elaboración de un cuestionario para evaluar la generación y gestión de residuos sanitarios en Institución de Enseñanza Superior. La preparación de este cuestionario se basa en las directrices del Consejo Nacional del Medio Ambiente y de la Agencia Nacional de Vigilancia Sanitaria, y también en las directrices de la Asociación Brasileña de Normas Técnicas y en los principios establecidos en la Política Nacional de Residuos Sólidos. El cuestionario incluye la generación y diferentes etapas de la gestión interna y externa de residuos, y ha sido construido con expertos y evaluado por jueces de la misma área. Se puede utilizar el cuestionario construido a partir de este estudio para hacer un diagnóstico inicial en las instituciones de enseñanza con el fin de verificar su situación en relación a residuos generados en sus límites. Este diagnóstico es crucial para ayudar en la preparación y seguimiento del Plan de Gestión de Residuos Sanitarios en Instituciones de Enseñanza y otros departamentos responsables por la generación de residuos sanitarios.

PALABRAS-CLAVE: Instituciones de Enseñanza Superior. Cuestionarios. Residuos Sanitarios.

\section{INTRODUÇÃO}

A intensificação da urbanização e o crescimento desordenado tem gerado a concentração das populações nas cidades, trazendo inúmeras dificuldades. Esse fator faz aumentar a diversidade e a complexidade dos problemas ambientais, demonstrando as fragilidades e contradições de um desenvolvimento industrial e tecnológico sem precedentes na história da humanidade (FERREIRA, 1995).

Nesse contexto, serviços essenciais podem deixar de ser oferecidos para a população devido à falta saneamento adequado que resulta na modificação e poluição do ambiente, expondo a população a situações de risco (SIQUEIRA; MORAES, 2009). Segundo a Organização Pan-Americana de Saúde (Opas), a maioria desses problemas tem repercussão direta na saúde e na qualidade de vida das pessoas (OPAS, 1999).

Em torno dessa problemática ambiental, surgem as discussões referentes ao conceito de saneamento ambiental que consiste em um conjunto de ações para buscar proporcionar ambientes salubres e habitáveis, sendo que uma das áreas prioritárias que vem causando grande preocupação diz respeito à gestão e 


\section{Periódica Eletranica \\ Fórum Ambiental}

da Alta Paulista
Volume 11, Número 08, 2015

Saúde e Saneamento Ambiental

gerenciamento de resíduos sólidos urbanos (RSU), e ao modo como são manejados, especialmente os resíduos perigosos. Entre esses resíduos, destacamse os resíduos de serviços de saúde (RSS), que, embora representem uma pequena quantidade da produção total de resíduos, oferecem risco à saúde e ao ambiente, pela possível presença de agentes químicos, biológicos e/ou radioativos (TAKAYANAGUI, 2005).

Os RSS podem apresentar características físicas e químicas, como: inflamabilidade, corrosividade, reatividade, toxicidade, patogenicidade, carcinogenicidade, teratogenicidade e mutagenicidade, enquadrando-se, nesse caso, como resíduos perigosos, classificados segundo a Associação Brasileira de Normas Técnicas como resíduos da Classe I (ABNT, 2004; BRASIL, 2010).

O despertar por interesse em estudos na área de resíduos não é recente (FERREIRA, 1995; JARDIM, 1998), e, atualmente, os RSS são alvo de grande preocupação devido a sua composição e seu potencial de periculosidade (PUGLIESI; GIL; SCHALCH, 2009), podendo causar sérios danos à saúde.

Emerge, assim, a necessidade de gerenciar, administrar e controlar a geração dos resíduos produzidos, devendo-se buscar uma otimização na estratégia integrada em seu manejo, combinando diferentes opções no gerenciamento (TCHOBANOGLOUS, 2009). A preocupação com a questão ambiental, social e econômica faz do gerenciamento de resíduos uma ferramenta importante no controle e na minimização do uso de recursos naturais, assim como, na promoção da saúde e na preservação e conservação do ambiente (BRASIL, 2006).

Nesse sentido, ao se incorporar os problemas decorrentes do gerenciamento inadequado de RSS, coloca-se a necessidade de desenvolver instrumentos capazes de avaliar as diferentes fases do gerenciamento desses resíduos, estabelecendo diretrizes de manejo na busca pela implementação de técnicas e tecnologias compatíveis com a realidade de cada instituição (GÜNTHER et al., 2010).

Atualmente, as diretrizes para o gerenciamento dos RSS são apresentadas na RDC 306/04 da Agência Nacional de Vigilância Sanitária (Anvisa) e na Resolução no 358/05 do Conselho Nacional do Meio Ambiente (Conama), divididos em cinco Grupos: A-Biológicos; B-Químicos; C-Radioativos; D-Comuns e E-Perfurocortantes (BRASIL, 2004, 2005), sendo esses resíduos de responsabilidade da instituição geradora, desde sua geração até a sua disposição final. Entre as fontes geradoras 
de RSS encontram-se as Instituições de Ensino Superior (IES), em especial os seus laboratórios de ensino, pesquisa e extensão, e outros serviços responsáveis pela geração de resíduos perigosos. Assim, essas instituições apresentam um papel fundamental, pois, apesar de sua importância em relação à produção de conhecimentos científicos, acabam, também, sendo fontes geradoras de resíduos de diferentes naturezas, muitos deles perigosos (PHILIPPI JR; AGUIAR, 2005).

Além disso, as IES devem buscar um desenvolvimento de forma sustentável que contribua para a disseminação de novos conceitos e práticas ao alcance de um processo de desenvolvimento em equilíbrio entre proteção ambiental, crescimento econômico e equidade social, sem comprometer a capacidade das gerações futuras de satisfazerem as suas próprias necessidades (CMMAD, 1991).

Estudos têm demonstrado a importância em desenvolver instrumentos de mensuração em diferentes áreas da saúde (LIMA, PORTELA, 2010; ROSA et al, 2008), mas em relação ao gerenciamento de resíduos de serviços de saúde, esse campo ainda é incipiente. Assim, a aplicação do questionário desenvolvido nesta pesquisa poderá auxiliar o setor administrativo das IES no levantamento de informações relevantes sobre a geração e o manejo dos seus resíduos para subsidiar a elaboração do Plano de Gerenciamento de Resíduos de Serviços de Saúde (PGRSS) que atenda às necessidades dos diferentes setores da instituição com as diretrizes que devem ser respeitadas em cada fase do manejo, de acordo com a legislação brasileira.

O presente estudo teve como objetivo elaborar e avaliar um questionário para auxiliar na organização de diagnóstico do gerenciamento de resíduos de serviços de saúde em Instituições de Ensino Superior.

\section{MATERIAL E MÉTODOS}

O delineamento deste estudo fundamentou-se na sistematização da literatura e análise da legislação vigente no Brasil acerca do gerenciamento de RSS. Para construção do questionário foram convidados especialistas que trabalham na área de resíduos biológicos, químicos, perfurocortantes, comuns e rejeitos radioativos, além de contar com a assessoria estatística e a participação de pesquisadores do 
Grupo Interinstitucional de Estudos da Problemática de Resíduos de Serviços de Saúde de Ribeirão Preto.

Nas primeiras reuniões foram discutidos, junto aos especialistas e pesquisadores da área de resíduos, os conceitos abordados na literatura atual, as diretrizes das resoluções $n^{\circ} 358 / 05$, do Conama e a RDC n`306/04 da Anvisa (BRASIL, 2004, 2005), da NBR 10.004/04 (ABNT, 2004) e os princípios presentes na Política Nacional de Resíduos Sólidos (PNRS) (BRASIL, 2010).

Foram considerados os princípios da legislação para fundamentar a construção das questões a partir da seleção de critérios e elementos correspondentes a cada fase do manejo dos resíduos. Esse procedimento foi baseado no objeto de investigação de acordo com os objetivos propostos para definir o universo do conteúdo, delineando suas divisões e subdivisões (FAYERS; MACHIN, 2007; PASQUALI, 2003), buscando traduzir em questões bem redigidas os objetivos da pesquisa (GIL, 2010).

Foi planejada, dessa forma, a base da estrutura de todas as questões abordadas no questionário a fim de fornecer informações que auxiliassem na elaboração do diagnóstico referente ao gerenciamento dos RSS. Critérios fundamentais como o conteúdo, tamanho, organização e clareza das questões, foram observados para estimular o informante a responder às questões abordadas (BARROS; LEHFELD, 1986). Assim, buscou-se na elaboração desse questionário uma organização concisa e de fácil compreensão dos respondentes, com a elaboração de questões semiestruturadas, de modo que o sujeito não necessitasse dispor de muito tempo para responder, sendo outra preocupação para obter maior colaboração e retorno dos sujeitos participantes.

Outro fator observado foi em relação ao formato e aparência que, também, podem influenciar significativamente no índice de retorno dos instrumentos (THOMAS; NELSON; SILVERMAN, 2007). Esses autores enfatizam, ainda, a importância da imparcialidade, devendo-se ter cuidado em não influenciar ou induzir o sujeito a responder às questões, cuidados esses, também, observados na construção do questionário. Todas as questões foram elaboradas deixando opções em aberto para que os sujeitos pudessem indicar alguma outra informação que julgassem importante acrescentar no levantamento. 
O questionário foi desenvolvido, inicialmente, para ser autoaplicado levando em consideração que a sua aplicação foi prevista junto a sujeitos responsáveis por laboratórios/serviços de IES com conhecimento técnico na área de gerenciamento de resíduos.

A partir dessa etapa da pesquisa, a participação de especialistas da área foi fundamental para a validação de face que se deve proceder na verificação da clareza dos tópicos e uma revisão crítica do instrumento (FAYERS; MACHIN, 2007). Os juízes foram convidados para avaliar o conteúdo do questionário, em termos do número e âmbito das questões, segundo os objetivos propostos.

Na seleção dos juízes foi utilizado como critério seu conhecimento científico e sua experiência prática no gerenciamento de resíduos de serviços de saúde, a fim de buscar uma contribuição de especialistas que atuassem na área de resíduos biológicos, químicos, comuns, perfurocortantes e rejeitos radioativos. Todos os especialistas receberam, previamente, uma carta convite e o Termo de Consentimento Livre e Esclarecido (TCLE) para participação na pesquisa, não tendo ocorrido recusa por parte dos especialistas convidados.

Os juízes avaliaram o conteúdo, a clareza, objetividade e organização do questionário. As sugestões e alterações foram analisadas e discutidas junto aos pesquisadores que participaram da construção inicial do questionário, para fazer as adaptações recomendadas antes de sua aplicação.

Este estudo foi previamente aprovado pelo Comitê de Ética em Pesquisa da Escola de Enfermagem de Ribeirão Preto da Universidade de São Paulo, em cumprimento às normas da Comissão Nacional de Ética em Pesquisa, presentes na Resolução do Conselho Nacional de Saúde no 196/96 (BRASIL, 1996).

\section{RESULTADOS E DISCUSSÃO}

Após a elaboração e avaliação qualitativa das questões pelos especialistas e juízes, foi produzido o questionário com questões referentes ao gerenciamento de RSS, que inclui conceitos do manejo interno e externo dos resíduos de diferentes Grupos: Resíduos Biológicos; Químicos; Comuns; Perfurocortantes e Rejeitos Radioativos (BRASIL, 2004, 2005). 
Não houve discrepância de conteúdo entre as sugestões dos juízes e discussões dos especialistas. As adaptações constituíram-se a substituições de alguns termos técnicos mais adequados ao estudo, mas que não alteravam o contexto da questão.

A partir dessa versão, foram sorteados 12 sujeitos responsáveis por serviços e laboratórios de ensino, pesquisa e extensão em Instituição de Ensino Superior a fim de verificar o tempo de resposta e entendimento do questionário. O tempo estimado de resposta foi de 15 minutos e não houve dificuldade de entendimento do questionário entre os sujeitos participantes.

Dessa forma, o questionário foi proposto em três itens com perguntas referente a identificação, geração e manejo dos resíduos. O primeiro item do questionário corresponde à identificação. Como o questionário foi desenvolvido para utilização em Instituições de Ensino Superior, foi solicitada a identificação da Unidade/Departamento, laboratório/serviço, nome do responsável, ramal, número da sala, setor, data, sendo, ainda, disponibilizado espaço para outras informações que o sujeito julgasse necessário. Ressalta-se que o item de identificação deve ser adequado para a realidade de cada instituição, segundo suas especificidades.

O segundo item corresponde a geração dos resíduos dos diferentes grupos, sendo disponibilizado algumas opções de cada tipo de resíduo segundo a legislação vigente e a periodicidade de sua geração na instituição. Optou-se por fazer um estudo preliminar a fim de selecionar algumas opções para facilitar o preenchimento do questionário, visando minimizar tempo e o índice de perdas. Vale ressaltar que em todas as questões o sujeito tinha a opção de indicar outras respostas que não estavam contempladas no questionário (Figura 1). 
Figura 1 - Questões do questionário referente aos tipos de resíduos gerados

II Quanto aos TIPOS de Resíduos gerados nesse Laboratório / Serviço:
Considerando a possibilidade de geração de diferentes TIPOS DE RESÍDUOS, por favor, assinale com um X, aqueles que são
produzidos neste Laboratório/Serviço, e indique a quantidade gerada SEMANALMENTE:

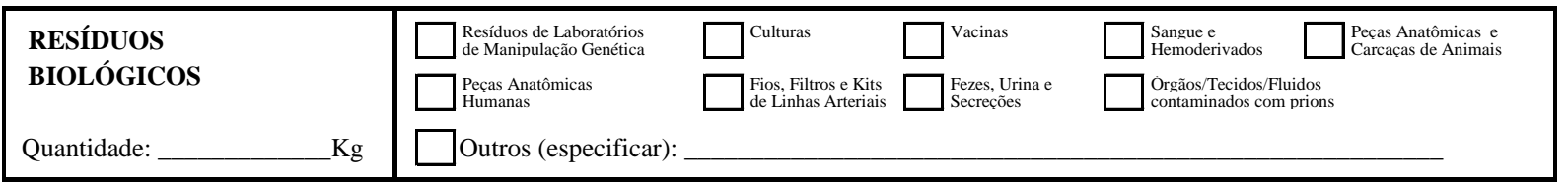

\begin{tabular}{|c|c|c|c|c|c|}
\hline RESÍDUOS & $\square^{\text {Acetona }}$ & Acetonitrila & Alcool & Benzeno & Clorofórmio \\
\hline QUÍMICOS & $\square^{\text {Eter }}$ & $\square^{\text {Hexano }}$ & Xilol & Brometo de Etídeo & $\begin{array}{l}\text { DAB } \\
\text { (diamina benzidina) }\end{array}$ \\
\hline & $\square$ Fenol & $\square^{\text {Formol }}$ & Corantes & Acidos & 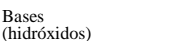 \\
\hline Volume: $\quad L$ & $\square$ Solucăo Sulfocrônica & Mercúrio & Prata & $\begin{array}{l}\text { Revelador/ } \\
\text { Fixador }\end{array}$ & $\begin{array}{l}\text { Medicamentos } \\
\text { Vencidos / Inutilizados }\end{array}$ \\
\hline Quantidade: __ $\quad K g$ & Outros (especificar): & & & & \\
\hline
\end{tabular}

\begin{tabular}{|l|llll|}
\hline REJEITOS & $\square{ }^{3} \mathrm{H}$ & $\square{ }^{32} \mathrm{P}$ & $\square^{125} \mathrm{I}$ & $\square{ }^{35} \mathrm{~S}$ \\
RADIOATIVOS & $\square^{51} \mathrm{Cr}$ & $\square{ }^{99} \mathrm{Tc}^{\mathrm{m}}$ & $\square{ }^{131} \mathrm{I}$ & $\square$ Timidinatritia \\
Quantidade: & $\mathrm{Kg}$ & $\square$ Outros (especificar): & & \\
\hline
\end{tabular}

\begin{tabular}{|c|c|c|c|c|c|}
\hline $\begin{array}{l}\text { RESÍDUOS } \\
\text { PERFUROCORTANTES } \\
\text { Quantidade: } \_ \text {Kg }\end{array}$ & $\begin{array}{l}\square^{\text {Agulhas }} \\
\square \text { Outros (especificar): }\end{array}$ & ]$_{\text {Similares }}^{\substack{\text { Bisturi e } \\
\text { Sin }}}$ & Brocas & $\begin{array}{l}\text { Lâminas, Limas } \\
\text { e Lamínulas }\end{array}$ & Vidraria \\
\hline Há contaminação desses & $\square$ Não & Não tenho & imento & & \\
\hline materiais com algum agente? & Agente: & Biológico & Químico & Radioativo & \\
\hline
\end{tabular}

\begin{tabular}{|c|c|c|c|}
\hline $\begin{array}{l}\text { RESÍDUOS COMUNS } \\
\text { (não contaminados) } \\
\text { Ouantidade: }\end{array}$ & $\begin{array}{l}\square \text { Orgânico } \\
\square_{\text {(näo contaminados) }} \text { OPapel } \\
\text { Outros (especificar): }\end{array}$ & Plástico $\square$ Metal & Vidro \\
\hline
\end{tabular}

O terceiro item do questionário proposto corresponde às diferentes fases do manejo dos resíduos. As questões foram separadas em manejo interno (segregação, acondicionamento, identificação, armazenamento, coleta, transporte e tratamento interno) e manejo externo (coleta, transporte, tratamento externo e disposição final) acrescentando-se algumas questões específicas, segundo sugestões dos pesquisadores e juízes, referentes à reutilização, reciclagem e descarte para complementar as informações sobre o gerenciamento dos resíduos no questionário para realização de um diagnóstico da geração/gerenciamento dos seus RSS.

A primeira questão desse item refere-se à segregação e ao acondicionamento dos resíduos (Figura 2). Na formulação dessa questão foram levadas em consideração as práticas mais frequentes nessas fases do manejo, separando-as nos diferentes grupos devido às particularidades apresentadas na legislação 
brasileira vigente (BRASIL, 2004, 2005). Destaca-se que os sujeitos poderiam indicar outras formas de realização de segregação e acondicionamento realizada em sua instituição.

Figura 2 - Questões do questionário referente a segregação e acondicionamento dos resíduos

\section{Quanto ao MANEJO dos Resíduos gerados:}

1 Por favor, assinale com um X, o modo como são SEGREGADOS e ACONDICIONADOS os resíduos logo após a geração, segundo seus diferentes tipos: (RESPONDER ESTA QUESTÃO, SOMENTE PARA OS RESÍDUOS INDICADOS NA QUESTÃO II ) SEGREGAÇÃO dos Resíduos BIOLÓGICOS: $\quad$ ACONDICIONAMENTO dos Resíduos BIOLÓGICOS:

\begin{tabular}{|l|l|}
\hline É realizada no local de sua geração & $\square$ São acondicionados em sacos plásticos da cor branca leitosa \\
\hline É realizada posteriormente & $\square$ São acondicionados em recipientes de plástico \\
$\square$ Não é realizada & $\square$ São acondicionados em caixas de papel resistente \\
$\square$ Não tenho conhecimento & $\square$ Outros: \\
\hline Outros: & $\square$
\end{tabular}

SEGREGAÇÃO dos Resíduos QUÍMICOS: ACONDICIONAMENTO dos Resíduos QUÍMICOS:

$\square$ É realizada no local de sua geração
$\square$ É realizada posteriormente
$\square$ Não é realizada
$\square$ Não tenho conhecimento
$\square$ Outros:

$\square$ São acondicionados em recipientes de vidro $\quad$ ( ) separadamente $\quad$ ( ) misturados
$\square$ São acondicionados em recipientes de plástico $($ ) separadamente $\quad$ ( ) misturados
$\square$ São acondicionados em recipientes de metal $\quad$ ( )separadamente $\quad$ ( ) misturados
$\square$ Não tenho conhecimento
$\square$ Outros:

SEGREGAÇÃO dos Rejeitos RADIOATIVOS: ACONDICIONAMENTO dos Rejeitos RADIOATIVOS:

$\square$ É realizada no local de sua geração
$\square$ É realizada posteriormente
$\square$ Não é realizada
$\square$ Não tenho conhecimento
$\square$ Outros:

SEGREGAÇÃO dos Res. PERFUROCORTANTES:

$\square$ São acondicionados em conteineres de chumbo
$\square$ São acondicionados em recipientes de vidro
$\square$ São acondicionados em recipientes de plástico
$\square$ Não tenho conhecimento
$\square$ Outros:

$\square$ É realizada no local de sua geração
$\square$ É realizada posteriormente
$\square$ Não é realizada
$\square$ Não tenho conhecimento
$\square$ Outros:

ACONDICIONAMENTO dos Resíduos PERFUROCORTANTES:

SEGREGAÇÃO dos Resíduos COMUNS:

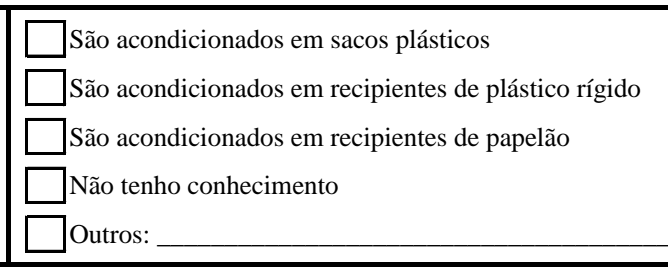

$\square$ É realizada no local de sua geração

ACONDICIONAMENTO dos Resíduos COMUNS:

$\square$ É realizada posteriormente

$\square$ Não é realizada

$\square$ Não tenho conhecimento

$\square$ Outros:

$\square$ São acondicionados em sacos plásticos
$\square$ São acondicionados em recipientes de plástico rígido
$\square$ São acondicionados em recipientes de papelão
$\square$ Não tenho conhecimento
$\square$ Outros:

$\square$ São acondicionados em sacos plásticos de cor preta

$\square$ São acondicionados em sacos plásticos de outra cor

$\square$ São acondicionados em caixas de papelão

$\square$ Não tenho conhecimento

$\square$ Outros: 
Na Figura 3, observam-se as demais questões referentes ao manejo interno dos resíduos (identificação, armazenamento, coleta, transporte e tratamento). Em algumas questões como coleta e transporte, podem ser adotados mais de uma forma de manejo segundo as observações dos sujeitos.

Figura 3 - Questões do questionário referente a identificação, armazenamento, coleta, transporte e tratamento interno dos resíduos

\section{Quanto à IDENTIFICAÇÃO:
$\square \operatorname{Sim}$
$\square$ Não
Não tenho conhecimento \\ Quanto ao ARMAZENAMENTO: \\ Existe neste Laboratório/Serviço um local exclusivo para armazenamento dos resíduos gerados? \\ $\square$ Sim; Especificar: \\ $\square$ Não; Especificar: \\ $\square$ Não tenho conhecimento \\ Quanto à COLETA INTERNA: \\ Quem é responsável pela coleta interna dos resíduos gerados neste Laboratório/Serviço? \\ $\square$ Funcionário de serviço Terceirizado \\ $\square$ Funcionário do quadro funcional da Instituição \\ $\square$ Não tenho conhecimento \\ $\square$ Outros (especificar):}

As embalagens utilizadas para o acondicionamento são identificadas pelo símbolo correspondente ao tipo de resíduo que contem?

Qual é a periodicidade com que é efetuada a coleta dos resíduos gerados neste Laboratório/Serviço?
$\square$ Diariamente
$\square$ Semanalmente
$\square$ Quinzenalmente
$\square$ Mensalmente
$\square$ Não tenho conhecimento
Outros (especificar):

\section{Quanto ao TRANSPORTE INTERNO:}

Qual é a forma utilizada para transportar os resíduos do local onde é gerado até onde é efetuada a coleta externa?

$\square$ Manual

$\square$ Carrinho sem tampa

$\square$ Carrinho com tampa

Não é efetuado o transporte interno: o resíduo é armazenado no próprio local de sua geração

$\square$ Não tenho conhecimento

$\square$ Outros (especificar):

\section{Quanto ao TRATAMENTO INTERNO:}

Os resíduos gerados neste Laboratório/Serviço recebem algum tipo de tratamento antes da coleta externa e/ou destinação final?
$\square \operatorname{Sim}$ $\square$ Não Não tenho conhecimento

Em caso afirmativo, indique o tipo de resíduo e seu respectivo tratamento:

Tipo de Resíduo:

Tratamento:

Tipo de Resíduo:

Tratamento:

Quanto ao manejo externo, o questionário apresenta questões referentes à coleta, transporte, tratamento e disposição final. Em relação ao tratamento externo, 
é proposto que as respostas sejam fornecidas segundo os diferentes grupos de resíduos (Figura 4), com a realização de uma análise prévia para adequar o questionário à realidade de instituições em futuros estudos.

Figura 4-Questões do questionário referente a etapas do manejo externo dos resíduos

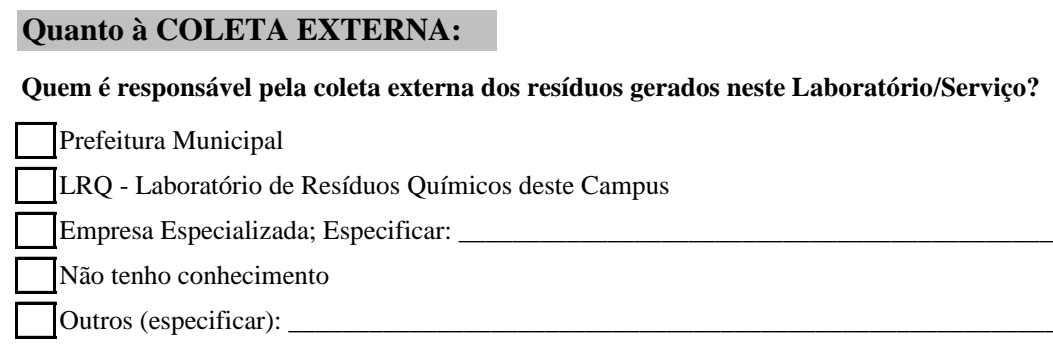

\section{Quanto ao TRANSPORTE EXTERNO:}

Qual é a forma utilizada para transportar os resíduos do local onde é coletado até o tratamento externo ou sua disposição final?

$\begin{array}{ll}\square \text { Veículo exclusivo para a coleta de resíduos especiais } & \square \text { Veículo para a coleta de recicláveis } \\ \square \text { Veículo para a coleta de resíduo comum } & \square \text { Não tenho conhecimento } \\ \square \text { Outros (especificar): } & \end{array}$

\section{Quanto ao TRATAMENTO EXTERNO:}

(RESPONDA ESTA QUESTÃO, SOMENTE PARA OS RESÍDUOS GERADOS NESSE LABORATÓRIO E INDICADOS NA QUESTÃO II )

Os resíduos gerados neste Laboratório/Serviço recebem algum tipo de tratamento externo?

$\square$ Sim $\square$ Não $\quad \square$ Não tenho conhecimento

Em caso afirmativo, indique o tratamento que cada tipo de resíduo gerado neste Laboratório/Serviço recebe:

\begin{tabular}{|lll}
\hline 1 - RESÍDUOS & $\square$ Autoclave $\quad \square$ Incineração & $\square$ Microondas $\square$ Desinfecção Química \\
BIOLÓGICOS & $\square$ Não tenho conhecimento & $\square$ Outros (especificar): \\
\hline
\end{tabular}

\begin{tabular}{|c|c|}
\hline $\begin{array}{c}2 \text { - RESÍDUOS } \\
\text { QUÍMICOS }\end{array}$ & $\square$ Incineração $\quad \square$ Neutralização/Destruição Química $\square$ Destilação/Recuperação \\
\hline Não tenho conhecimento & $\square$ Outros (especificar): \\
\hline
\end{tabular}

\begin{tabular}{|lll}
\hline 3- REJEITOS & $\square$ Decaimento da Meia Vida \\
RADIOATIVOS & $\square$ Não tenho conhecimento $\quad \square$ Outros (especificar): \\
\hline
\end{tabular}

\begin{tabular}{|lll}
\hline 4- RESÍDUOS & $\square$ Desinfecção Química & $\square$ Incineração \\
PERFUROCORTANTES & $\square$ Não tenho conhecimento & $\square$ Outros (especificar):
\end{tabular}

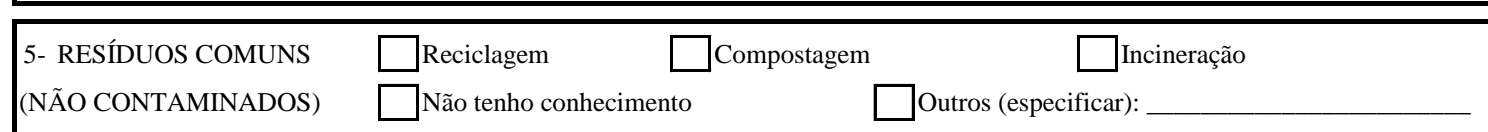

\section{Quanto à DESTINAÇÃO FINAL:}

Qual é o tipo de destinação final dada aos resíduos gerados neste Laboratório/Serviço?
$\square$ Aterro Sanitário
$\square$ Aterro Industrial
$\square$ Vala Séptica
$\square$ Dispostos diretamente no solo (no Campus)
$\square$ Queimados (no Campus)
$\square$ Não tenho conhecimento

$\square$ Outros (especificar): 
Segundo sugestão dos especialistas que auxiliaram na construção do instrumento e dos juizes que participaram de sua avaliação, ainda foram elaboradas questões referentes à reutilização, reciclagem e descartes dos resíduos/rejeitos. A Figura 5 contem a apresentação dessas questões, fundamentadas nas diretrizes apresentadas na Política Nacional dos Resíduos Sólidos (BRASIL, 2010).

Figura 5 - Questões do questionário referente a reutilização, reciclagem e descarte dos resíduos

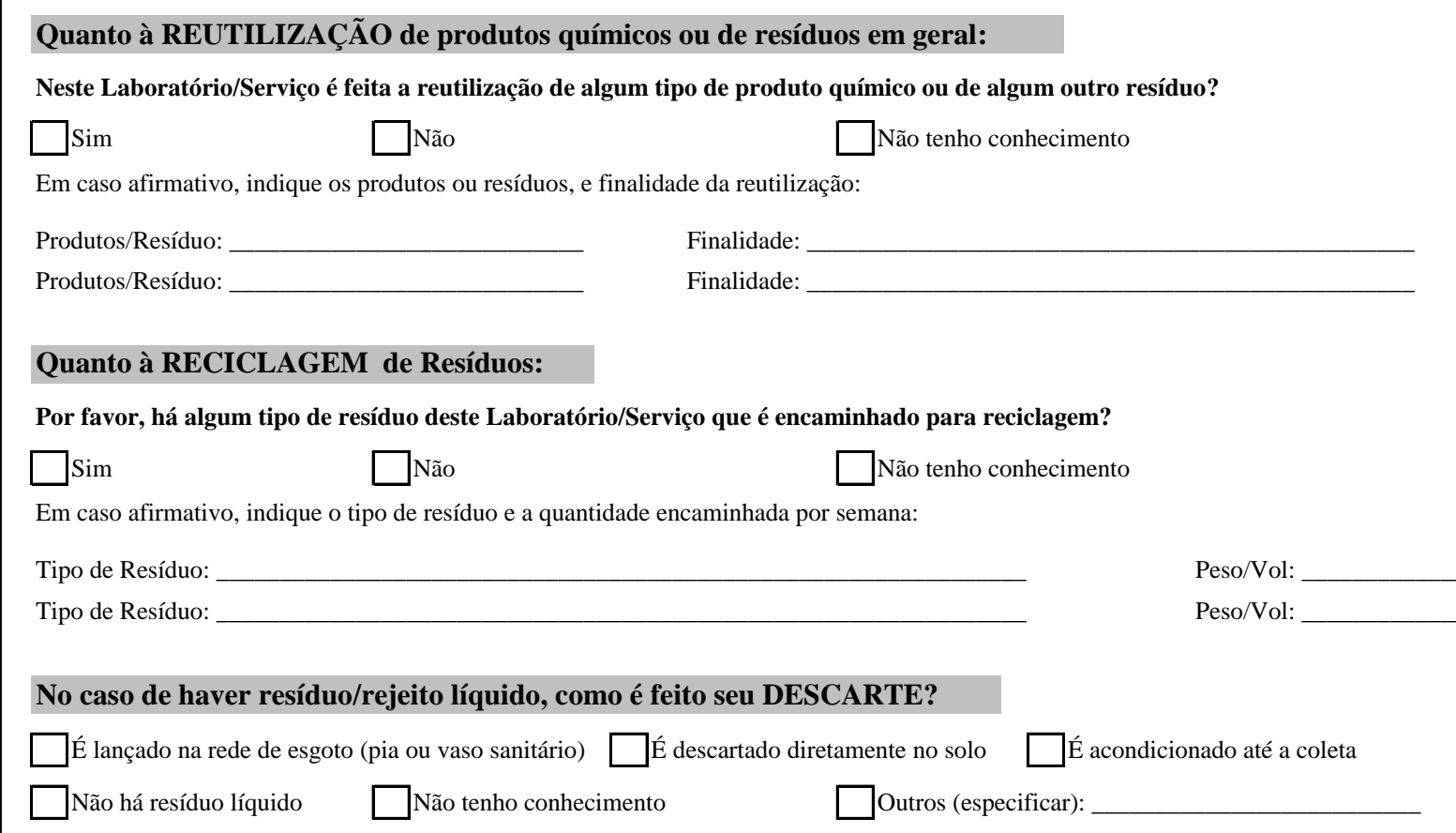

\section{CONCLUSÕES}

A produção científica na área de gerenciamento de RSS tem obtido grandes avanços nos últimos anos. Contudo, o aumento de geração e a diversidade desses resíduos impõe a necessidade de investimentos contínuos nessa área. As IES como fontes geradoras de conhecimento devem ser o espelho para a sociedade, buscando soluções sustentáveis no gerenciamento dos resíduos gerados em suas delimitações.

O questionário desenvolvido, a partir deste estudo, pode ser uma ferramenta importante nesse processo, fornecendo subsídios para a realização de diagnósticos preliminares referente a geração/gerenciamento dos RSS em IES. Essas 


\section{Periódica Eletrânica \\ Fórum Ambiental}

da Alta Paulista
Volume 11, Número 08, 2015

Saúde e Saneamento Ambiental

informações são essências para a elaboração do PGRSS, proporcionando a adequação das instituições junto às legislações brasileiras vigentes, reforçando o seu comprometimento social e ambiental perante a sociedade.

O questionário foi desenvolvido para ser autoaplicado, considerando que essa metodologia para coleta de dados permite uma abrangência de um maior número de sujeitos com uma economia de tempo. Contudo, devem ser adotados os procedimentos para que possa permanecer a confiabilidade e imparcialidade em sua aplicação.

Visando esses princípios, o questionário foi construído indicando algumas opções fundamentadas nas legislações brasileiras a fim de facilitar seu preenchimento. Porém, foi garantida, em todas as questões, a possibilidade do sujeito acrescentar outra informação que não foi abordada, possibilitando uma participação mais ativa.

Embora esse questionário tenha sido desenvolvido com uma visão voltada a IES, espera-se que ele possa fundamentar novos estudos na área, respeitando as adequações e validações necessárias para aplicação em outros serviços responsáveis por gerar RSS. Em realidades com um menor número de sujeitos e maior diversidade no grau de instrução, que pode resultar na dificuldade de seu preenchimento, o questionário pode ser aplicado de forma presencial. Nesse caso, os entrevistadores devem ser devidamente treinados para evitar possíveis intervenções junto aos sujeitos.

Cabe ressaltar, ainda, a importância da entrega de uma carta explicativa e o termo de consentimento junto ao questionário, a fim de esclarecer possíveis dúvidas, os objetivos e uma breve descrição da pesquisa, para que os sujeitos tenham ciência do propósito do estudo e da importância de seu comprometimento, buscando maior participação dos sujeitos.

Acredita-se que o questionário construído, a partir desta pesquisa, possa auxiliar na realização de novos estudos na área de avaliação diagnóstica de Gerenciamento de Resíduos de Serviços de Saúde em diferentes Instituições/Serviços da sociedade. Todavia, existem particularidades nos diferentes setores na área de saúde responsáveis por gerar esse tipo de resíduo, em que todo e qualquer acompanhamento deve ser realizado por profissionais com habilitação técnica na área. 


\section{Periódica Eletranica ISSN 1980-0827 \\ Fórum Ambiental \\ da Alta Paulista \\ Volume 11, Número 08, 2015 \\ Saúde e Saneamento Ambiental}

Os diagnósticos obtidos a partir da aplicação do questionário apresentam um perfil inicial da situação do gerenciamento dos RSS nas Instituições/Serviços, fornecendo informações essenciais aos gestores das instituições para fundamentar a elaboração, acompanhamento e avaliação de seu PGRSS.

Diagnósticos nessa área podem, também, propiciar um estímulo ao desenvolvimento de políticas públicas para um maior controle das técnicas/procedimentos que devem ser adotados no gerenciamento de resíduos dos diferentes grupos, considerando a variedade e especificidade dos resíduos que são encontradas nessas instituições.

Espera-se que outras instituições à luz de uma preocupação ambiental, econômica e social, possam utilizar o questionário elaborado como parâmetro para a realização de novos estudos e levantamentos que possam despertar um olhar crítico dos dirigentes das instituições.

\section{Agradecimentos}

Esta pesquisa foi desenvolvida com o apoio financeiro do Conselho Nacional de Desenvolvimento Científico e Tecnológico (CNPq).

\section{REFERÊNCIAS}

[ABNT] Associação Brasileira de Normas Técnicas. NBR 10.004: Resíduos sólidos - Classificação. Rio de Janeiro: Associação Brasileira de Normas Técnicas; 2004.

BARROS, Aidil Jesus Paes; LEHFELD, Neide Aparecida Souza. Fundamentos de metodologia: um guia para a inicialização científica. São Paulo: McGraw-Hill; 1986.

BRASIL. Lei no 12.305, de 02 de agosto de 2010. Institui a Política Nacional de Resíduos Sólidos. Diário Oficial da União. Brasília; 2010.

Ministério da Saúde. Manual de gerenciamento de resíduos de serviço de saúde. Brasília: Ministério da Saúde; 2006.

Conselho Nacional do Meio Ambiente. Resolução $n^{\circ}$ 358. Dispõe sobre o tratamento e a disposição final dos resíduos de saúde e dá outras providências. Diário Oficial da União. Brasília; 2005.

Agência Nacional de Vigilância Sanitária. Resolução RDC n 306, de 07 de dezembro de 2004. Dispõe sobre o regulamento técnico para o gerenciamento de resíduos de serviços de saúde. Diário Oficial da União. Brasília; 2004.

. Resolução no 196/96. Diretrizes e normas regulamentadoras sobre pesquisa envolvendo seres humanos. Conselho Nacional de Saúde. Brasília; 1996. 


\section{Periódica Eletrônica

[CMMAD] Comissão Mundial sobre Meio Ambiente e Desenvolvimento. Nosso futuro comum. Rio de Janeiro: Editora Fundação Getúlio Vargas; 1991.

FAYERS, Peter; MACHIN, David. Scores and Measurements: Validity, Reliability, Sensitivity. In: FAYERS, Peter; MACHIN, David. Quality of life: the assessment, analysis, and interpretation of patient-reported outcomes. 2nd ed. England: John Wiley \& Sons Ltd; 2007. p. 77-108

FERREIRA, João Alberto. Resíduos Sólidos e Lixo Hospitalar: Uma Discussão Ética. Caderno Saúde Pública 1995; 11:314-20.

GIL, Antonio Carlos. Como elaborar projetos de pesquisa. 5nd ed. São Paulo: Atlas; 2010.

GÜNTHER, Wanda Maria Risso et al. Elaboração de plano de gerenciamento de resíduos de serviço de saúde - PGRSS. São Paulo: Universidade de São Paulo; 2010.

JARDIM, Wilson Figueiredo. Gerenciamento de resíduos químicos em laboratórios de ensino e pesquisa. Química Nova, São Paulo, v. 21, n. 5, p. 671-73. 1998.

LIMA, Maria José Barbosa; PORTELA, Margareth Crisóstomo. Elaboração e Avaliação da confiabilidade de um instrumento para medição da qualidade de vida relacionada à saúde de idosos independentes. Caderno Saúde Pública, Rio de Janeiro, v. 26, p. 1651-62. 2010.

[OPAS] Organização Pan-americana de saúde. Atenção primária ambiental. Brasília: Gráfica e Editora Brasil; 1999.

PASQUALI, Luiz. Psicometria: teoria dos testes na psicologia e na educação. Petrópolis: Editora Vozes; 2003.

PHILIPPI JR, Arlindo; AGUIAR, Alexandre Oliveira. Resíduos sólidos: características e gerenciamento. In: PHILIPPI JR; Arlindo, organizador. Saneamento, saúde e ambiente: fundamentos para um desenvolvimento sustentável. São Paulo: Manole; 2005. p. 267-321

PUGLIESI, Erica; GIL, Tatiana Novis Lopes; SCHALCH, Valdir. Caracterização qualitativa e quantitativa dos resíduos de serviço de saúde gerados em hospital de médio porte no município de São Carlos, SP. Revista Minerva, São Carlos, v. 6, p. 213-17. 2009.

ROSA, Marcos Aurélio Camargo; SLAVUTZKY, Sonia Maria Blauth; PECHANSKY, Flávio; KESSLER, Félix. Processo de desenvolvimento de um questionário para avaliação de abuso e dependência de açúcar. Caderno Saúde Pública, Rio de Janeiro, v. 24, p. 1869-76. 2008.

SIQUEIRA, Mônica Maria; MORAES, Maria Silvia. Saúde coletiva, resíduos sólidos urbanos e os catadores de lixo. Ciência Saúde Coletiva, v. 14, p. 2115-22. 2009.

TAKAYANAGUI, Angela Maria Magosso. Gerenciamento de resíduos de serviços de saúde. In: PHILIPPI JR, Arlindo, organizador. Saneamento, saúde e ambiente: fundamentos para um desenvolvimento sustentável. São Paulo: Manole, 2005; p. 323-74

TCHOBANOGLOUS, George. Solid Waste Management. In: NEMEROW, Nelson; AGARDY, Franklin; SULLIVAN, Patrick, SALVATO, Joseph. Environmental engineering: environmental health and safety for municipal infrastructure, land use and planning, and industry. 6 ed. New Jersey: Wiley, 2009; p. 177-308.

THOMAS, Jerry; NELSON, Jack; SILVERMAN, Stephen. Métodos de pesquisa em atividade física. SALES, Denise Regina; DORNELLES, Márcia Santos. Tradução. Porto Alegre: Artmed; 2007. 